\title{
BMJ Open Determining the effects of transcranial direct current stimulation on tinnitus and tinnitus-related outcomes: protocol for a systematic review
}

\author{
Bas Labree (D) ,,2 Derek J Hoare (D) , , Lauren E Gascoyne (D) , ${ }^{3}$ \\ Magdalena Sereda (D) ${ }^{1,2}$
}

To cite: Labree B, Hoare DJ, Gascoyne LE, et al. Determining the effects of transcranial direct current stimulation on tinnitus and tinnitus-related outcomes: protocol for a systematic review. BMJ Open 2021;11:e047191. doi:10.1136/ bmjopen-2020-047191

- Prepublication history and supplemental material for this paper is available online. To view these files, please visit the journal online (http://dx.doi. org/10.1136/bmjopen-2020047191).

Received 23 November 2020 Revised 08 February 2021 Accepted 10 March 2021

A) Check for updates

C Author(s) (or their employer(s)) 2021. Re-use permitted under CC BY-NC. No commercial re-use. See rights and permissions. Published by BMJ.

${ }^{1}$ NIHR Nottingham Biomedical Research Centre, Nottingham, UK

${ }^{2}$ Hearing Sciences,

Mental Health and Clinical Neuroscience, University of Nottingham, Nottingham, UK ${ }^{3}$ Sir Peter Mansfield Imaging Centre, School of Physics and Astronomy, University of Nottingham, Nottingham, UK

Correspondence to

Bas Labree;

bas.labree@nottingham.ac.uk

\section{ABSTRACT}

Introduction Tinnitus is the awareness of a sound in the ear or head in the absence of an external source. It affects around $10 \%-15 \%$ of people. About $20 \%$ of people with tinnitus also experience symptoms such as depression or anxiety that negatively affect their life. Transcranial direct current stimulation (tDCS) is a technique involving constant low-intensity direct current delivered via electrodes on the head. It is postulated to modulate (suppress or enhance) neural activity in the region between electrodes. As such, it represents a potential treatment option for tinnitus, as well as comorbid depression or anxiety. This systematic review will estimate the effects of tDCS on outcomes relevant to tinnitus. In addition, it will determine whether there is any relationship between stimulation parameters (electrode montage, current intensity, and length and frequency of stimulation sessions) and the effect of tDCS on these outcomes.

Methods and analysis Electronic searches for peerreviewed journal articles will be performed in the Cochrane Register of Studies online (the Cochrane Ear, Nose and Throat Disorders Group Register and CENTRAL, current issue), PubMed, EMBASE, CINAHL, LILACS, KoreaMed, IndMed, PakMediNet, CNKI, AMED, PsycINFO, Web of Science, ClinicalTrials.gov, ICTRP and Google Scholar using the following search terms: transcranial Direct Current Stimulation OR tDCS AND tinnitus OR depression $O R$ anxiety $O R$ quality of life $O R$ adverse effects $O R$ neurophys*

Searches were not limited by date. Methods are reported according to the Preferred Reporting Items for Systematic Reviews and Meta-Analyses Protocols (PRISMA-P). Randomised controlled trials will be included if they report at least one of the following outcomes: tinnitus symptom severity, anxiety or depression as measured by relevant validated instruments. Where available, data on quality of life, adverse effects and neurophysiological changes will also be reviewed. In addition to an analysis of the effect of each parameter, an analysis will be performed to uncover any interactions between parameters. Where appropriate, meta-analyses will be performed.

Ethics and dissemination This systematic review will make use of secondary data only. As no data will be obtained from participants directly, ethical approval has not been sought. No other ethical issues are foreseen. Findings will be submitted for peer-reviewed publication
Strengths and limitations of this study

- This review will provide a meticulous overview of the existing evidence on the effects of transcranial direct current stimulation (tDCS) on tinnitus symptom severity, depression and anxiety.

- The protocol allows for the inclusion of all trials in which the effects of tDCS on any of these outcomes were measured in people with any health condition, even if these were not primary outcomes.

- Furthermore, it will be the first systematic review investigating the relationship between stimulation parameters (electrode montage, current intensity, stimulation duration and number of stimulation sessions) and the effects of tDCS.

- This review will only include trials using tDCS, excluding high-definition tDCS. Therefore, no comparisons between neuromodulation techniques will be conducted.

and presented at academic conferences. The results of this review will inform future research.

PROSPERO registration number CRD42020185567.

\section{INTRODUCTION}

Tinnitus is the perception of sound, commonly described as a ringing, buzzing or hissing, in the absence of an external sound source. ${ }^{1}$ The perceived location of the tinnitus varies, but most often it is perceived in the ear(s) or head. It is estimated that between $10 \%$ and $15 \%$ of adults experience chronic tinnitus (lasting longer than 3 months), while $20 \%$ of those have clinically significant symptoms. ${ }^{2} \mathrm{~A}$ particular association exists between tinnitus and mood disorders. A recent systematic review found a high prevalence of depression in individuals with tinnitus. ${ }^{3}$ In the absence of a clear mechanism by which tinnitus and depression interact, the authors proposed three possible associations: (1) depression affecting tinnitus, (2) tinnitus predisposing 
its sufferers to depression and (3) tinnitus appearing as a comorbidity with depression. A subsequent population study with a large sample $(\mathrm{n}=21.4$ million respondents with tinnitus) which sought to quantify the relationship between tinnitus, depression and anxiety found further evidence for this relationship. In this study, $26.1 \%$ of tinnitus sufferers but only $9.2 \%$ of controls reported symptoms of anxiety in the previous year. A strikingly similar pattern was observed in depression; $25.6 \%$ of tinnitus sufferers but only $9.1 \%$ of controls reported symptoms of depression. Furthermore, those reporting tinnitus as a 'big' or 'very big' problem were more likely to report anxiety and depression. ${ }^{4}$

Current clinical management strategies include psychological interventions-including cognitive-behavioural therapy-education, sound therapy and combinations thereof such as tinnitus retraining therapy. However, the effectiveness of these interventions is variable. Several experimental treatments for tinnitus have been proposed, such as pharmacological interventions, ${ }^{5-8}$ dietary supplementation $^{9}$ and neuromodulation treatments. ${ }^{10}$ One experimental treatment in the last category is transcranial direct current stimulation (tDCS), a neuromodulation technique that involves the application of a weak electrical current $(0.5-2 \mathrm{~mA})$ to the cortex via electrodes applied to the scalp. The key mechanism involved is the subthreshold modulation of neuronal membrane potentials. This mechanism leads to changes in cortical excitability and neuronal activity. ${ }^{11}$ Rather than induce neural activity directly, tDCS is thought to increase or decrease the likelihood of action potentials in a neural population, through this change in excitability. Investigators of many disorders which are at least partially underpinned by abnormal neurophysiological states have used tDCS both as a tool to better understand these disorders and as a potential treatment. The treatment rationale is that, if maladaptive neural activity can be inhibited and normal activity restored, this may result in an improvement of symptoms, particularly if this leads to long-lasting neuroplastic changes. ${ }^{12} 13$

tDCS has most extensively been used for depression (for reviews, see previous works ${ }^{13-16}$ ). Major depressive disorder is usually accompanied by changes in cortical excitability which are thought to reflect dysfunction at the cortical and subcortical level. ${ }^{16}$ As tDCS is understood to cause changes in cortical excitability, many studies on tDCS and depression investigate whether this can be used to alter depression-related non-typical excitability in a way that ameliorates the symptoms of depression. Studies primarily interested in other conditions sometimes include a measure of generalised depression as the disorder being investigated may lead to symptoms of depression (eg, see previous works ${ }^{17}{ }^{18}$ ). Anxiety is another condition for which tDCS has been investigated as a possible treatment. ${ }^{19}$ Similar to depression, anxiety disorders are accompanied by an abnormal neurophysiological state. The use of tDCS for anxiety similarly targets cortical excitability, with the aim of reducing symptoms.
Ultimately, the goal of any intervention to improve symptoms is to bring about an improvement in patients' quality of life. Therefore, some tDCS studies include a measure of general or health-related quality of life (for an example, see previous work ${ }^{20}$ ). If $\mathrm{tDCS}$ is effective in improving quality of life, this would likely be a result of the change in symptom severity resulting from the tDCS, rather than being a direct result of the stimulation.

Given the putative mechanisms of tDCS, a measure of neurophysiological change associated with the application of tDCS is of interest. Such a measure can shed further light on the neurophysiological underpinnings of any significant effect on outcomes. Neurophysiological measures such as magnetoencephalography (MEG) and electroencephalography (EEG) have been used to investigate neurophysiological change brought about by tDCS. Neurophysiological measures may provide insights into the effects of tDCS on particular outcomes, or the mechanisms of these effects. ${ }^{21}$ For this reason, it has become increasingly common for a measure of neurophysiological change to be included in tDCS studies.

Adverse effects of tDCS are relevant to any use of the technique as safety and tolerability may affect the feasibility of its clinical application. Known adverse effects of tDCS include a tingling, burning or itching sensation, and redness of the skin at the stimulation sites. Studies typically report no adverse effects at all, or report mild, transient adverse effects, although there may be selective reporting. A systematic review of the reporting and assessment of adverse effects in tDCS studies found that of the 209 studies included, only 56\% reported the presence or absence of adverse effects. ${ }^{22}$ It is vital for clinical studies to report adverse effects as the feasibility of $\mathrm{tDCS}$ as an intervention for any condition depends on it being safe and well-tolerated by patients. For this reason, investigating the effects of stimulation parameters such as electrode montage, current intensity, stimulation duration and the number of stimulation sessions on safety and tolerability is just as important as investigating the effect of these parameters on effectiveness.

Across studies of tDCS for tinnitus, stimulation parameters used (such as electrode montage, current intensity, duration and frequency of stimulation sessions) vary widely. Currently, there is no consensus on the optimal setup for improvements in tinnitus symptom severity. Nevertheless, there is emerging evidence for the effectiveness of tDCS for tinnitus. For instance, one review and meta-analysis concluded that while limited evidence was available, active tDCS was associated with a better treatment outcome than sham. ${ }^{23}$ The existing evidence suggests that differences in stimulation parameters can lead to different outcomes. Anodal tDCS over the left temporoparietal area with the cathode over the contralateral frontal scalp has been observed to result in a transient suppression of tinnitus in some participants. ${ }^{24} 25$ There is also some evidence of a polarity-specific effect on tinnitus loudness suppression when the auditory cortex is targeted ${ }^{26} \mathrm{~A}$ trial investigating the effect of 
multiple sessions of tDCS over the left temporoparietal area, however, found no effect on tinnitus symptoms. ${ }^{27}$ Another configuration, bifrontal tDCS, targets the dorsolateral prefrontal cortex (with the anodal electrode over EEG 10-20 location F3 and the cathodal over F4, or vice versa) and has been shown to result in temporary suppression of tinnitus perception and tinnitus distress. ${ }^{28-34} \mathrm{~A}$ trial comparing electrode montages targeting the left temporoparietal area and the dorsolateral prefrontal cortex (as well as an HD-tDCS set-up) found similar clinical outcomes for each configuration. ${ }^{35}$ There is also emerging evidence of an improvement in tinnitus symptoms following multiple sessions of bifrontal tDCS for tinnitus: one study found that an increase in the number of sessions-up to six, after which a plateau occurredled to a reduction in tinnitus loudness. ${ }^{36}$ Two randomised controlled trials (RCTs) have found a significant beneficial effect on tinnitus symptom severity. ${ }^{37} 38$ This effect was not observed in isolation; however, improvements in depression and anxiety were also found in one of these trials. ${ }^{37}$

In terms of neurophysiological change (ie, change in function as measured by EEG or MEG), a recent randomised clinical trial combined tDCS and EEG and reported an improvement in tinnitus symptoms as well as a modulation of cortical electrical activity. Tinnitus annoyance and severity were significantly improved and a correlated decrease in beta, theta and alpha frequency oscillations were observed. Standardised low-resolution brain electromagnetic tomography (sLORETA) implicated the frontal, temporoparietal and limbic regions in these changes in frequency bands. Improvements in tinnitus severity and annoyance were associated with decreased beta and theta EEG frequency bands while participants' eyes were open, and decreased alpha frequency when they were closed. Changes in frequency bands in the frontal, temporoparietal and limbic regions were found using sLORETA and negative correlations were observed between baseline EEG frequency bands and tDCS-induced change in tinnitus annoyance and severity. ${ }^{39}$ MEG has also been used to investigate oscillatory neural activity in tinnitus. One prominent finding from MEG studies of tinnitus is a possible association between slow-wave oscillatory activity and tinnitus. ${ }^{40} 41$ One mechanism by which tDCS might therefore affect tinnitus symptoms is by interacting with the oscillatory activity associated with tinnitus.

This systematic review will assess the effects of tDCS on tinnitus symptom severity, depression and anxiety. Where reported, we will also assess the effects of tDCS on quality of life and neurophysiological change, and associated adverse effects. The relationship between stimulation parameters and these effects will also be reviewed.

\section{METHODS AND ANALYSES}

This protocol is reported according to the Preferred Reporting Items for Systematic Reviews and Meta-Analyses
Protocols (PRISMA-P) ${ }^{42}$ and has been registered on PROSPERO (registration number: CRD42020185567).

\section{Databases and search strategy}

Electronic searches for peer-reviewed journal articles will be performed in the Cochrane Register of Studies online (the Cochrane Ear, Nose and Throat Disorders Group Register and CENTRAL, current issue), PubMed, EMBASE, CINAHL, LILACS, KoreaMed, IndMed, PakMediNet, CNKI, AMED, PsycINFO, Web of Science, ClinicalTrials.gov, ICTRP and Google Scholar using the following search terms: transcranial Direct Current Stimulation OR tDCS AND tinnitus OR depression OR anxiety OR quality of life OR adverse effects OR neurophys*.

An example of the full search strategy as used when searching PubMed can be found in online supplemental information file 1 .

The reference lists of identified publications will be hand-searched for additional records. In addition, PubMed, TRIPdatabase, The Cochrane Library and Google will be searched to retrieve published systematic reviews relevant to this systematic review, so that their reference lists can be hand-searched for additional records. Searches were not limited by date.

\section{ELIGIBILITY CRITERIA}

Published or in press peer-reviewed journal articles reporting RCTs or cross-over trials (eligible if data from before the crossover can be extracted, to avoid the potential for a carry-over phenomenon) will be included. Only records available in English will be included, as there are no resources available for translation. There will be no restriction on year of publication or publication status. Study authors may be contacted for clarification or to request data where trial reports are incomplete or not available. Inclusion criteria have been specified according to PICO (Participants, Intervention, Comparator, Outcome(s)).

\section{Participants}

Participants are 18 years of age or older, with any health condition.

\section{Intervention}

At least one session of tDCS using any electrode montage and any stimulation parameters. Studies where the intervention was high definition tDCS will be excluded.

\section{Comparator}

Sham (placebo) tDCS, no intervention, waiting list control.

\section{Primary outcomes}

- Tinnitus symptom severity, as measured by the global score on a multi-item tinnitus questionnaire or visual analogue scales.

- Generalised anxiety, as measured by the validated instrument. 
- Generalised depression, as measured by the validated instrument.

\section{Secondary outcomes}

Where available, data on the following outcomes will also be extracted and analysed.

- Quality of life.

- Adverse effects.

- Neurophysiological changes (as measured, eg, with EEG or MEG).

\section{Screening}

BL, MS, DH and LG will screen all studies retrieved to determine their eligibility for inclusion based on information from the title and abstract, with each record being screened by two authors independently. Included records will then be screened based on full text. Again, each record will be screened by two authors independently. Any disagreements between authors will be discussed until a consensus is reached, with a third author arbitrating if necessary.

\section{Data extraction}

BL, MS, DH and LG will extract data using a purposefully designed data extraction form, with all data being extracted by two authors independently. The data extraction form will be piloted on a subset of articles and revised if required before formal data extraction begins. After data extraction is complete, the data extracted by each author will be compared for accuracy and consistency. Any discrepancies between authors will be discussed until a consensus is reached, with a third author arbitrating if necessary.

Information to be extracted will include trial design, setting, methods of randomisation and blinding, power, inclusion and exclusion criteria, stimulation parameters (electrode montage, current intensity, stimulation duration and number of stimulation sessions) and control, treatment fidelity, type and duration of follow-up, and outcome measures and statistical tests; baseline characteristics of participants (demographic information, data on the primary and secondary outcome measures), attrition or exclusion, dropout rates, adverse effects, outcome group mean and SD at pre-intervention and post-intervention and follow-up, statistical tests and results of between-group comparisons.

The authors will be contacted where further information is required that is not contained within the trial publication or in an accessible database. If not reported or provided by the authors, SD will be estimated using the available data such as SEs, CIs, $p$ and t values. Where data are only available in graph form, authors will approximate numerical data using semiautomatic software developed for this purpose (https://automeris.io/ WebPlotDigitizer/).

\section{Quality assessment}

Two authors will independently assess risk of bias of the included RCTs, with the following taken into consideration, as guided by the Cochrane Handbook for Systematic Reviews of Interventions ${ }^{43}$ :

- Sequence generation.

- Allocation concealment.

- Blinding.

- Incomplete outcome data.

- Selective outcome reporting.

- Other sources of bias.

Disagreements between authors will be discussed until a consensus is reached, with a third author arbitrating if necessary.

\section{Descriptive analysis and meta-analysis \\ Data synthesis}

Data synthesis will be performed using RevMan V.5.4.1. ${ }^{44}$ Outcomes will be analysed separately. If more than one study is identified for a given outcome, and if combining studies is appropriate, meta-analyses will be performed. If not, a narrative synthesis will be conducted. Data from RCTs will be pooled using a fixed-effect model, except when heterogeneity of aggregated effect sizes is found. This will be calculated using Cochran's $Q$ statistic $\left(\chi^{2}\right.$ test with K-1 degrees of freedom, with $\mathrm{K}$ being the number of studies) and the $I^{2}$ statistic (with percentages of approximately $25 \%, 50 \%$ and $75 \%$ of $I^{2}$ being interpreted as low, medium and high heterogeneity, respectively). ${ }^{45}$ Dichotomous data will be pooled using the RR measure, while continuous data will be pooled using the standardised mean difference measure, if more than one tool is used to measure the same outcome. The psychometric properties of questionnaires will be considered with regard to their suitability for pooling. Data will only be included from multi-item questionnaires that show similar responsiveness and can be assumed to measure the same underlying construct (high convergent validity) as other multi-item questionnaires for the same outcome. The authors will independently examine the information available for each instrument, and categorise the instruments for potential inclusion in meta-analyses. Risk of bias will be assessed using the Cochrane Collaboration's tool for assessing risk of bias. ${ }^{44}$ Studies assessed as having a high risk of bias will be excluded.

\section{Sensitivity analyses}

Studies included in the review will be further analysed according to the parameters they used (electrode montage, current intensity, stimulation duration and number of stimulation sessions). This will allow us to examine whether the effects of the stimulation is affected by these parameters.

\section{Patient and public involvement}

This review is part of a PhD project which has been reviewed by the National Institute for Health Research Nottingham Biomedical Research Centre Patient and Public Involvement group.

Amendments

None to date. 


\section{Dissemination}

Registered information will be updated as the review progresses. On completion, this review will be submitted for publication in a peer-reviewed journal and presented at national and international scientific conferences.

\section{DISCUSSION}

While tDCS may represent a promising treatment approach for tinnitus, there are many uncertainties and confounds that need to be untangled. Beneficial effects on tDCS on tinnitus symptom severity are often observed alongside improvements in depression and anxiety. ${ }^{37}$ The exact relationship between these outcomes is unclear. This review will assess the effects of tDCS on outcomes relevant for tinnitus, including tinnitus symptom severity, depression and anxiety. Where available, data on quality of life, neurophysiology and adverse effects will be collated. It will also determine from the existing evidence whether electrode montage, current intensity, stimulation duration and the number of stimulation sessions affect the effects of tDCS on these outcomes. Together, these analyses will inform hypotheses on what tDCS protocol is likely effective for tinnitus and/or common tinnitus-related symptoms. The main strength of this review lies in its scope. In addition to including tinnitus symptom severity, depression and anxiety, the inclusion of neurophysiological change will allow for insight into whether there is evidence for an underlying mechanism. The inclusion of measures of adverse effects will allow for the assessment of safety and tolerability of tDCS as a potential treatment for tinnitus, and of whether safety and tolerability differs depending on different parameters used. Furthermore, the inclusion of RCTs investigating any health condition means that data on the effects of tDCS on tinnitus symptom severity, depression and anxiety that have previously been excluded from different reviews because of their focus on a specific condition will now be included in a single review, resulting in a more complete overview of the existing evidence. This review will also assess the effects of particular parameters on the efficacy of tDCS for tinnitus and tinnitus-related outcomes. Depending on the findings of the review, this evidence could form a solid basis for the design of future clinical trials, in particular development of the stimulation protocol optimised for tinnitus.

Twitter Derek J Hoare @Derek_J_Hoare, Lauren E Gascoyne @DrGascoyne and Magdalena Sereda @MagdalenaSereda

Contributors All authors devised the protocol. BL drafted the manuscript. DJH, LEG and MS provided critical feedback on the manuscript. All authors reviewed and approved the final manuscript for submission.

Funding BL, DJH and MS are funded through the National Institute for Health Research (NIHR) Biomedical Research Centre programme. LEG is funded by the UK Quantum Technologies Hub. The views expressed are those of the authors and not necessarily those of the NIHR, the NHS, the Department of Health and Social Care, or the UK Quantum Technologies Programme.

Competing interests None declared.

Patient consent for publication Not required.
Provenance and peer review Not commissioned; externally peer reviewed.

Supplemental material This content has been supplied by the author(s). It has not been vetted by BMJ Publishing Group Limited (BMJ) and may not have been peer-reviewed. Any opinions or recommendations discussed are solely those of the author(s) and are not endorsed by BMJ. BMJ disclaims all liability and responsibility arising from any reliance placed on the content. Where the content includes any translated material, BMJ does not warrant the accuracy and reliability of the translations (including but not limited to local regulations, clinical guidelines, terminology, drug names and drug dosages), and is not responsible for any error and/or omissions arising from translation and adaptation or otherwise.

Open access This is an open access article distributed in accordance with the Creative Commons Attribution Non Commercial (CC BY-NC 4.0) license, which permits others to distribute, remix, adapt, build upon this work non-commercially, and license their derivative works on different terms, provided the original work is properly cited, appropriate credit is given, any changes made indicated, and the use is non-commercial. See: http://creativecommons.org/licenses/by-nc/4.0/.

\section{ORCID iDs}

Bas Labree http://orcid.org/0000-0002-3999-4562

Derek J Hoare http://orcid.org/0000-0002-8768-1392

Lauren E Gascoyne http://orcid.org/0000-0002-0825-0587

Magdalena Sereda http://orcid.org/0000-0002-2792-8496

\section{REFERENCES}

1 Jastreboff PJ. Phantom auditory perception (tinnitus): mechanisms of generation and perception. Neurosci Res 1990;8:221-54.

2 Baguley D, McFerran D, Hall D. Tinnitus. The Lancet 2013;382:1600-7.

3 Geocze L, Mucci S, Abranches DC, et al. Systematic review on the evidences of an association between tinnitus and depression. Braz J Otorhinolaryngol 2013;79:106-11.

4 Bhatt JM, Bhattacharyya N, Lin HW. Relationships between tinnitus and the prevalence of anxiety and depression. Laryngoscope 2017;127:466-9.

5 Baldo P, Doree C, Molin P, et al. Antidepressants for patients with tinnitus. Cochrane Database Syst Rev 2012;30.

6 Hilton MP, Zimmermann EF, Hunt WT, et al. Ginkgo biloba for tinnitus. Cochrane Database Syst Rev 2013;322.

7 Hoekstra CEL, Rynja SP, van Zanten GA, et al. Anticonvulsants for tinnitus. Cochrane Database Syst Rev 2011;265.

8 Wegner I, Hall DA, Smit AL, et al. Betahistine for tinnitus. Cochrane Database Syst Rev 2018;12:CD013093.

9 Person OC, Puga ME, da Silva EM, et al. Zinc supplementation for tinnitus. Cochrane Database Syst Rev 2016;11:CD009832.

10 Hoare DJ, Whitham D, Henry JA, et al. Neuromodulation (desynchronisation) for tinnitus in adults. Cochrane Database Syst Rev 2015;29.

11 Woods AJ, Antal A, Bikson M, et al. A technical guide to tDCS, and related non-invasive brain stimulation tools. Clin Neurophysiol 2016;127:1031-48.

12 Lefaucheur J-P, Antal A, Ayache SS, et al. Evidence-Based guidelines on the therapeutic use of transcranial direct current stimulation (tDCS). Clin Neurophysiol 2017;128:56-92.

13 Brunoni AR, Ferrucci R, Fregni F, et al. Transcranial direct current stimulation for the treatment of major depressive disorder: a summary of preclinical, clinical and translational findings. Prog Neuropsychopharmacol Biol Psychiatry 2012;39:9-16.

14 Berlim MT, Van den Eynde F, Daskalakis ZJ. Clinical utility of transcranial direct current stimulation (tDCS) for treating major depression: a systematic review and meta-analysis of randomized, double-blind and sham-controlled trials. J Psychiatr Res 2013;47:1-7.

15 Meron D, Hedger N, Garner M, et al. Transcranial direct current stimulation (tDCS) in the treatment of depression: systematic review and meta-analysis of efficacy and tolerability. Neurosci Biobehav Rev 2015;57:46-62.

16 Nitsche MA, Boggio PS, Fregni F, et al. Treatment of depression with transcranial direct current stimulation (tDCS): a review. Exp Neurol 2009;219:14-19.

17 Pal N, Maire R, Stephan MA, et al. Transcranial direct current stimulation for the treatment of chronic tinnitus: a randomized controlled study. Brain Stimul 2015;8:1101-7.

18 Palm U, Keeser D, Hasan A, et al. Prefrontal transcranial direct current stimulation for treatment of schizophrenia with predominant 
negative symptoms: a double-blind, sham-controlled proof-ofconcept study. Schizophr Bull 2016;42:1253-61.

19 Vicario CM, Salehinejad MA, Felmingham K, et al. A systematic review on the therapeutic effectiveness of non-invasive brain stimulation for the treatment of anxiety disorders. Neuroscience \& Biobehavioral Reviews 2019;96:219-31.

20 Mori $\mathrm{F}$, Codecà $\mathrm{C}$, Kusayanagi $\mathrm{H}$, et al. Effects of anodal transcranial direct current stimulation on chronic neuropathic pain in patients with multiple sclerosis. J Pain 2010;11:436-42.

21 Mangia AL, Pirini M, Cappello A. Transcranial direct current stimulation and power spectral parameters: a tDCS/EEG coregistration study. Front Hum Neurosci 2014;8:601.

22 Brunoni AR, Amadera J, Berbel B, et al. A systematic review on reporting and assessment of adverse effects associated with transcranial direct current stimulation. Int J Neuropsychopharmacol 2011;14:1133-45.

23 Song J, Vanneste S, Van de Heyning P. Transcranial direct current stimulation in tinnitus patients: a systematic review and metaanalysis. Sci World J 2012.

24 Garin P, Gilain C, Damme J-P, Van Damme J, et al. Short- and long-lasting tinnitus relief induced by transcranial direct current stimulation. J Neurol 2011;258:1940-8.

25 Shekhawat GS, Stinear CM, Searchfield GD. Transcranial direct current stimulation intensity and duration effects on tinnitus suppression. Neurorehabil Neural Repair 2013;27:164-72.

26 Joos K, De Ridder D, Van de Heyning P, et al. Polarity specific suppression effects of transcranial direct current stimulation for tinnitus. Neural Plast 2014;2014:1-8.

27 Forogh B, Mirshaki Z, Raissi GR, et al. Repeated sessions of transcranial direct current stimulation for treatment of chronic subjective tinnitus: a pilot randomized controlled trial. Neurol Sci 2016;37:253-9.

28 Vanneste S, Plazier M, Ost J, et al. Bilateral dorsolateral prefrontal cortex modulation for tinnitus by transcranial direct current stimulation: a preliminary clinical study. Exp Brain Res 2010;202:779-85.

29 Vanneste S, van de Heyning P, De Ridder D. The neural network of phantom sound changes over time: a comparison between recentonset and chronic tinnitus patients. Eur J Neurosci 2011;34:718-31.

30 Vanneste S, Walsh V, Van De Heyning P, et al. Comparing immediate transient tinnitus suppression using tACS and tDCS: a placebocontrolled study. Exp Brain Res 2013;226:25-31.

31 Vanneste S, De Ridder D. Bifrontal transcranial direct current stimulation modulates tinnitus intensity and tinnitus-distress-related brain activity. Eur J Neurosci 2011;34:605-14.
32 De Ridder D, Vanneste S. Eeg driven tDCS versus Bifrontal tDCS for tinnitus. Front Psychiatry 2012;3:84.

33 Faber M, Vanneste S, Fregni F, et al. Top down prefrontal affective modulation of tinnitus with multiple sessions of tDCS of dorsolateral prefrontal cortex. Brain Stimul 2012;5:492-8.

34 Frank E, Schecklmann M, Landgrebe M, et al. Treatment of chronic tinnitus with repeated sessions of prefrontal transcranial direct current stimulation: outcomes from an open-label pilot study. $J$ Neurol 2012;259:327-33.

35 Jacquemin L, Shekhawat GS, Van de Heyning P, et al. Effects of electrical stimulation in tinnitus patients: conventional versus highdefinition tDCS. Neurorehabil Neural Repair 2018;32:714-23.

36 Shekhawat GS, Vanneste S. Optimization of transcranial direct current stimulation of dorsolateral prefrontal cortex for tinnitus: a non-linear dose-response effect. Sci Rep 2018;8:1-8.

37 Yadollahpour A, Bayat A, Rashidi S, et al. Dataset of acute repeated sessions of bifrontal transcranial direct current stimulation for treatment of intractable tinnitus: a randomized controlled trial. Data Brief 2017;15:40-6.

38 To WT, Ost J, Hart J, et al. The added value of auditory cortex transcranial random noise stimulation (tRNS) after bifrontal transcranial direct current stimulation (tDCS) for tinnitus. J Neural Transm 2017; 124:79-88.

39 Souza DdaS, Almeida AA, Andrade SMdosS, Andrade S, et al. Transcranial direct current stimulation improves tinnitus perception and modulates cortical electrical activity in patients with tinnitus: a randomized clinical trial. Neurophysiol Clin 2020;50:289-300.

40 Adjamian P, Sereda M, Hall DA. The mechanisms of tinnitus: perspectives from human functional neuroimaging. Hear Res 2009;253:15-31.

41 Adjamian P, Sereda M, Zobay O, et al. Neuromagnetic indicators of tinnitus and tinnitus masking in patients with and without hearing loss. JARO 2012;13:715-31.

42 Moher D, Shamseer L, Clarke M, et al. Preferred reporting items for systematic review and meta-analysis protocols (PRISMA-P) 2015 statement. Syst Rev 2015;4:1.

43 Higgins J, Green S. Cochrane Handbook for systematic reviews of interventions version 5.1.0. The Cochrane Collaboration.

44 RevMan 5.4.1 the Cochrane collaboration 2020.

45 Huedo-Medina TB, Sánchez-Meca J, Marín-Martínez F, et al. Assessing heterogeneity in meta-analysis: $Q$ statistic or $\mathrm{I}^{2}$ index? Psychol Methods 2006;11:193-206. 